\title{
EMERGENT PROCESSES AS GENERATION OF DISCONTINUITIES
}

Published in: G. Minati, E. Pessa, M. Abrams (eds.) (2012). Methods, Models, Simulations and Approaches Towards a General Theory of Change. Singapore: World Scientific, 135-146.

\author{
Leonardo Bich, Gianluca Bocchi \\ Dipartimento di Scienze della Persona and Post-Graduate School of \\ Anthropology and Epistemology of Complexity, University of Bergamo, P.le \\ S.Agostino 2, 24129 Bergamo, Italy \\ E-mail: leobich@libero.it
}

In this article we analyse the problem of emergence in its diachronic dimension. In other words, we intend to deal with the generation of novelties in natural processes. Our approach aims at integrating some insights coming from Whitehead's Philosophy of the Process with the epistemological framework developed by the "autopoietic" tradition. Our thesis is that the emergence of new entities and rules of interaction (new "fields of relatedness") requires the development of discontinuous models of change. From this standpoint natural evolution can be conceived as a succession of emergences - each one realizing a novel "extended" present, described by distinct models - rather than as a single and continuous dynamics. This theoretical and epistemological framework is particularly suitable to the investigation of the origin of life, an emblematic example of this kind of processes.

Keywords: diachronic emergence, constructivism, hierarchy theory, non-algorithmic processes, origin of life.

"Order is not sufficient. What is required is something much more complex. It is order entering upon novelty; so that the massiveness of order does not 
degenerate into mere repetition; and so that the novelty is always reflected upon a background of system." (A. N. Whitehead)

\section{Introduction: Emergence from Identity to Process}

One of the wider and more radical controversies that characterize contemporary thought - which however has a long tradition in the modern age and even before in the ancient one - opposes some views which consider a material or energetic foundation as primary in the natural world - whereby the processes of evolution and development would have only a derivative or even epiphenomenic status - and some other views which, on the contrary, consider the dimension of development and creativity as primary and fundamental. ${ }^{1}$ The first kind of approaches leads to a reductionist practice, inclined to trace the complex forms of organization back to the properties of single and disconnected elements. ${ }^{2,3}$ The second identifies a continuous creative process in which systems of great complexity emerge ${ }^{4}$ as an ubiquitous feature of our universe. This controversy concerns the implicit theoretical and epistemological basic assumptions about the generative potential of the world: the idea of a creative nature able to give rise to qualitatively new forms is thus opposed to a view of natural world as a mere rearrangement of elements already and forever defined and fixed.

The idea of emergence plays a crucial role for every approach that stresses the inherent creativity of the universe. Emergence, however, is a wide concept: a label under which many phenomena, models, descriptive relations can be collected. It can express: part-whole relations; different kinds of descriptive limits; the constitution of systems from basic components; the expression of new behaviors and properties; creative historical processes etc.

In The Structure of Science $e^{5}$ Nagel introduces the topic of emergence by criticizing the generic use of the expression "emergent evolution". He asserts that it confuses two different concepts that would need to be carefully distinguished: the first is a thesis on hierarchical organizations, specifically on the predictability or not of higher level properties in terms of lower level ones; the second one is a cornerstone of a evolutionary cosmology, a thesis concerning the "creative advance of nature" which 
would give rise to novel entities or properties. The latter version of emergence, according to him, is not entailed by the former. Other authors, instead, propose weaker distinctions. ${ }^{6}$

Any attempt to develop an emergentist approach to the problem of change needs to take into consideration this issue. According to us, there is no need to establish a-priori a strong distinction between the two versions of the concept: focusing on either synchronic (hierarchical) or diachronic (processual) aspects, however, can be useful and even necessary for specific descriptive purposes. In our view the concept of emergence concerns, in a very broad sense, the appearing of qualitative novelties in the natural world, and it entails a specific thesis in the characterization of this novelty: the un-deducibility of the new features of the world - a higher level entity as well as new behaviors or properties - from an initial and predetermined model, which can consist in the description of the basic component as well as the description of the initial state in a historical dynamics. From this point of view, the two meanings of emergence strongly separated by Nagel should be revalued as two dimensions of the same issue, concerning the limits in our capabilities, as scientific observers, of interacting with complex natural phenomena: the insufficiency of a single modality of description and the necessity to recur to multiple irreducible ones, as an inescapable aspects of our relationship with the world ${ }^{7,8,9}$. The sub-classifications, thus, should be always functional to the main focus of the investigation, whether it is directed to the definition of the identity of a system or to the characterization of a historical process. What shouldn't be ignored, as we will show in the following sections, is that hierarchical and processual aspects are strictly intertwined.

According to these remarks is it possible to re-formulate the distinction between synchronic and diachronic emergence as two perspectives on the same issue:

- Synchronic emergence concerns the recognition by the observer of a unitary system, the characterization of its identity as not deducible from its components, and the identification of different levels of analysis. The focus of the investigation is mainly the relation between pars and whole. An example is the model of a living system. 
- Diachronic emergence concerns the processes that give rise to new entities, properties and behaviors in the natural world, not deducible from the initial ones. The level of description is that of the interaction between systems and their environments. Examples are the models of the origin of life and of the processes of speciation.

In previous articles ${ }^{10,11}$ the synchronic perspective has been developed, while the purpose here is to analyse what idea of process is entailed by the definition of emergence given above. Our analysis is theoretical and epistemological, rather than metaphysical. It is placed at the level of the interface between the observer and the natural world. Consequently it is focused on the modalities of access to reality and on the conceptual models of change that derive from the dialogical interaction between subject and object. ${ }^{12,13,9,14}$

\section{Modes of Change}

The basic idea of diachronic emergence consists in the impossibility to deduce the future steps of a process from even the most accurate description of the present and past ones. In order to understand the implications of this descriptive limit, it is necessary to start by considering what can change in the description of a temporal dynamics, and then pointing out the differences between a reductionist approach and an emergentist one. With respect to these aspects, the notion of logical openness ${ }^{15,16,17}$ can play a useful role in expressing how the possibilities of modeling a system change according the complexity of its internal dynamics and of its interactions with the environment. To the increase in the degree of logical openness of a system corresponds its higher ability to express different dynamics and behaviors from those predicted by individual models.

The simplest form of change is the succession of states, which exhibits the form of an input-output relation: given some rules of transformation, boundary conditions, and some initial value (input) of the variable under study, after a certain amount of time the variable will assume a new value (output). This form of dynamics, of course, does not produce any effective novelty: everything is already implicitly given at 
the beginning. According to the framework proposed by the theory of logical openness, ${ }^{15,16,17}$ it corresponds to a level of openness zero.

In order to observe some effective change taking place in the dynamics it is necessary to focus not just on states, but on those factors — or "informational levels" 18 — of our descriptive models of a system and of system/environment interactions, which contribute to define the dynamics itself:

(i) The rules of interaction;

(ii) The boundary conditions;

(iii) The parameters of the system.

The difference between a model of a process that admits effective novelty and one which does not, lies in the capability or not to define and modify these three factors ${ }^{18,9}$. It is sufficient to think of the radical difference between an object sliding along an inclined plane and the internal environment generated by a living system. In the first case the rules of interaction are fixed as well as the properties of the object, and the boundary conditions are external and not influenced by the dynamics: all the three aspects are extrinsic to each other and not modified by the process they take part into. As we said above, in this case the logical openness of the model of the system under study is zero, as the system does not behave in a different way from that described by the initial model.

Things are different when these informational levels are not strictly separated, but in mutual interaction in such a way that the levels of logical openness increase. In the second case - concerning the internal environment of an organism - in fact, the internal environment is selfrealized and self-maintained, and in certain degrees can be changed. The constitution of a living system separated from its surrounding environment, establishes a new internal context, so introducing - at least some - new boundary conditions for its internal dynamics (metabolism). The metabolism produces new kinds of components that behave in different ways, with the result of defining new rules of interaction that, in turn, can change some of the properties of the internal context ${ }^{9,19,20,12,18,8}$. In this case the three informational levels are not extrinsic to each other and independent, but are co-defined and, as a consequence, their change requires the elaboration of new models. In 
other words, the action of a system on its own definitory constraints specifying its internal dynamics as well as its modalities of interaction with the environment - increase its logical openness, so establishing limits in principle in the possibility of modeling it.

This modality of change can take place only under specific conditions at the level of the basic theoretical assumptions, conditions that are not satisfied in a typical mechanistic or reductionist framework. The latter is based, in fact, on the theses that (a) all the entities involved in a dynamics can be exhaustively characterized by their intrinsic properties; (b) that the environment is fixed and cannot be influenced by the objects that interact in it; and (c) that the rules that describe these interactions are external - that is - the interactions do not change the properties of the entities that interact and, vice versa, the modalities of interaction are not modified by these same objects. ${ }^{9}$ As Whitehead - among the others pointed out ${ }^{21,22,23,24}$, this is not necessarily a characteristic of reality in itself. Rather, it is just a possible perspective, which consist in the result of a procedure of theoretical construction, that in the reductionist framework, however, is assumed as an ontological foundation (the fallacy of misplaced concreteness): an abstraction from the integrated unity of the natural world (mechanism of extensive abstraction) that leads to define objects in themselves without reference to nothing else (principle of simple localization of matter). The starting point of the reductionist approach is, thus, the definition of what is "fundamental": self-sufficient entities with extrinsic interactions. Then, this basic framework is extended to the all the levels of the natural world. The result is that everything is already given at this fundamental (selfsufficient) level and all changes in the synchronic and diachronic dimensions are consequently epiphenomenic. ${ }^{25}$

The independency of informational levels and entities, on the one hand, can be useful in the modeling or control of simple systems. Here the form of change "input then output" or " $\delta \mathrm{A}$ then $\delta \mathrm{B}$ " implies that what changes in one factor of the description does not influence the others, or that the interaction between elements does not change or define some of their properties. On the other hand it fails (a) in describing the dynamics of integrated and transformative - or better, complex ${ }^{7,18,8}$ systems like living ones and (b) in accounting for effective processes 
able to generate any kind of novelty in the natural world, beside a mere rearrangement of elements.

\section{Systemic Hierarchies}

From the theoretical point of view the mechanistic framework has, as a result, the construction of inclusive hierarchies of sets, like Simon's modules $^{26}$ or Jacob's intregrones". They consist in "Chinese-box" constructions that share three main properties. (i) The first is that, when a certain domain of investigation is approached, in principle it is always possible to find an "ultimate" or basic model pertaining what is considered in this case as the fundamental level. All the information necessary in order to describe the system is in principle already present at this lower level. Also, the entities that are relevant in it are the result of a specific operation. They are, in fact, characterized as the material, or structural parts ${ }^{10,11}$ through their intrinsic properties - that is - those properties that are independent of their relational context: describing them in isolation or in interaction does not make any difference. Then the higher hierarchical level under investigation can be conceptually built from these basic bricks. (ii) From this follows the second property. It consists in the possibility to fractionate the higher level entities without loss of information. The main reason is that the elements are defined without reference to anything else, and the interactions that give rise to bigger aggregates are not transformative of their properties. (iii) The third consists in the possibility to ascribe some form of functional autonomy to higher order entities. Nonetheless it is just a useful heuristic operation that allows to abstract from some descriptive steps. It does not exclude that everything necessary for the description of the phenomenon under study is in principle already present at the fundamental level: higher ones are ultimately epiphenomenic and derivable from it.

The most celebrated example of this foundational and reductionist perspective is the concept of physicalism ${ }^{3,28}$, which claims that in the world there is nothing but the "physical", meaning with that the "basic physics", whatever it could be. Yet, the idea of basic self-sufficient entities or particles has been deeply questioned in microphysics, ${ }^{25,29,30}$ the same domain to which the status of fundamental is ascribed by 
reductionist approaches. The main criticism consists in pointing out how different theoretical operations can lead to the characterization of entities with different characteristics. On the basis this argument, a different interpretation of the notion of particle has been proposed: from the basic brick of reality to a theoretical construct useful as a heuristic tool. ${ }^{30}$

In order to account for the creativity of nature and the properties of complex systems a different path is to be followed. The alternative consists in abandoning the idea of a fundamental level of description, and in focusing on the modalities of access to the natural world ${ }^{25,11}$. It means rejecting the theoretical procedure that starts from the bottom of a hierarchy, ascribes to it an ontological primary status, and then builds all the others levels from it. The main specificity of this approach consists, rather, in taking into consideration another possible observational operation besides the identification of the only material parts of a system. Depending on the descriptive purposes of the observer and the properties of the chosen domain of investigation, this operation is the distinction of those entities which are relevant on that domain, a distinction that is made with respect to the context these entities realize and that at the same time makes them possible. They are the "functional components" of the object of study, which are characterized through their relational properties ${ }^{11}$ and require what can be labeled as a mesoscopic approach to function. They constitute meta-structures ${ }^{31}$ that need to be characterized top-down and that give rise to a distinct domain of description with respect to the one established by the aggregation of material parts. As such, they can require different modalities of description with respect to structural components (e.g. relational models ${ }^{8}$ ).

The result of this approach is that at any descriptive level the pertinent entities and their context are co-defined, co-specified and coconstructed. In such a framework there is not a primary level but all are related to the operations of the observer: they are transcendental rather than ontological ${ }^{25}$. Therefore, it is possible to conceive some novelty in the higher levels. They can be considered - using an expression coined by Lloyd Morgan ${ }^{32}$ - as new fields of relatedness, in which terms and relations co-emerge and are co-dependent.

The hypothesis of integrative systemic hierarchies has an important tradition. Examples can be found in Feibleman's Theory of Integrative 
Levels $^{33}$, Anokhin's Theory of Functional Systems ${ }^{34,35}$ and in more recent formulations like Baas' Hyperstructures ${ }^{36}$. All share the idea of intertwined levels, where the relation between bottom-up (starting from basic level) and top-down (starting from higher level) description is not trivial - that is - it is not easy to establish a strict correspondence between structural (anatomic or material parts) components and functional ones.

As we pointed out elsewhere, ${ }^{9,10,11}$ in Rosen's idea of a noncorrespondence between structure and function, and between synthesis and analysis, ${ }^{8}$ lies a possible interpretation of emergence as undeducibility. In fact, if higher levels are emergent and, thus, not deducible from lower ones, the description of the basic components (the material parts) is not sufficient in order to model them. Consequently, in order to describe the higher level dynamics, new components functional ones - need to be identified in the emergent relational domain. And, under the undeducibility condition, these new functional components and the material ones realize two different meta-structures whose models do not coincide.

From a diachronic point of view the consequences of this hierarchical emergent framework are twofold. (a) The first is that the levels are strictly co-dependent in both directions, bottom-up and top-down, not just in the first one: there is no reason to consider any relevant change as taking place effectively only at the basic level. Thus, by observing a dynamics on a meta-level of description which takes into consideration more levels at the same time, it is possible to identify correspondences - mutual specifications - between changes at different levels or descriptive domains. ${ }^{9}$ Similarly — in Bitbol's "interventionistconstitutive view" 29 - we can conceive that an intervention that operates some changes on one level can trigger modifications at other levels.

(b) The second consequence is the aspect of novelty brought forth by diachronic emergence. The emergence of an integrative level, in the form of a new systemic unity, establishes a novel dynamics: new boundary conditions, new rules of interactions (relations) and new elements - that is - new functional components that allow its modeling. It is the previous example of the separation between the internal and external environment brought forth by the emergence of the living. But the 
novelty does not concern only the underlining dynamics of an integrated unity. In fact, a new entity introduces new relational properties at the higher level of its interaction with the environment and, for this reason, it contributes to the modification of this more comprehensive dynamics. An example can be the emergence of life and its influence on the early earth environment; another the birth of a new species that gives rise to a new "functional component", a new niche, in the dynamics of the more comprehensive ecological system, so redefining its field of relatedness. In these cases new structural and functional meta-structures establish new levels of interaction that generate new system/environment constraints and, in doing so, new levels of logical openness.

\section{A Model of Diachronic Emergence as a Succession of Fields of Relatedness}

From the theoretical and epistemological point of view, the concept of emergent systemic hierarchies with functional components expresses an idea that is similar in some aspects to that introduced by Whitehead in metaphysics through the concept of concrescence ${ }^{22}$ : a web in which every element influences and is influenced by the others in the very definitory properties. This idea implies that the engine of the generation of novelty is the appearing of new elements: an entity that emerges synchronically modifies its context.

It is on this aspect of "novelty that contributes to novelty" at different levels of description, that depends the kind of undeducibility that specifically characterizes diachronic emergence. In fact, if elements, dynamics and context are co-defined at any level or descriptive domain and are not built starting from a fundamental level, then any change in one of the three factors of this domain triggers a reorganization of it: new functional components and new dynamics. Therefore, the new behavior of the domain under investigation is no more deducible from the rules which were pertinent before the reorganization.

This form of change constitutes a challenge to our capability of modeling, as it imposes strong limitations in the possibility of derivation of new processual steps from the previous ones. ${ }^{19}$ As Kauffman asserts, in fact, the novel laws that emerge in the historical process are similar to 
the introduction of new axioms and - we say - new rules of transformation, from which new consequences can be derived ${ }^{20}$.

Then, what view of change is entailed by this framework? In the first place it introduces a strong version of novelty that is not to be considered just as something that was not there before. It is grounded, instead, on the condition of diachronic un-deducibility. In the second place, from the point of view of our descriptions it has consequences that are similar to those of synchronic emergence, but on the horizontal dimension. In fact, if a behavior, a property or a dynamics cannot be derived from its antecedent, but requires the formulation of new models, we are facing some sort of diachronic fragmentation in the domain under investigation. In such a perspective the whole process can be considered as a succession of relational emergent discontinuities ${ }^{9}$.

We are used to some sort of discontinuity in the synchronic dimension between the physical, biological, cognitive and social domains especially in the debate about emergence. Yet the importance of discontinuity has been acknowledged also in natural processes, both in cosmology and in evolutionary theory. ${ }^{37,38,39,40,20,4}$ From the point of view of emergence as undeducibility, discontinuity needs to be interpreted not just as a difference in the rhythms of transformation, opposed to a gradualist view, but as a consequence of the generation of new entities and levels of organization which play an effective role in re-defining the dynamics in which they are involved. Therefore, we characterize it not as a problem in the time scales of our models, but as a form of "fragmentation" of the domain of their temporal validity.

What emerges at every creative step of a natural process is, therefore, a "novel present", with a new field of relatedness that requires specific models in order to be described. This implication is particularly evident in the biological domain, as Maturana and Mpodozis assert: "the living system does not encounter a preexisting niche because it appears with its living, and it does not see or relate to a preexisting medium because it does not encounter the medium beyond that which appears in the realization of its niche. [...] Both in the ontogeny and the phylogeny of living systems, living system and niche change together, and [...] indeed, living system and medium change together in a continuous becoming of 
the biosphere as an interconnected network of living and not living systems that operationally arises at every instant as a novel present". ${ }^{41}$

This novel present can be described as "extended" or "epochal". By this we mean that certain duration can be ascribed to it. This duration is not constant for any epochal present and its extent can be defined on the basis of the validity of our models or - in other words - on the basis of the interval of invariance of the informational levels that characterize them and their degree of logical openness.

What a novel present is depends on the characteristic of the domain of investigation and on the hierarchical level observed. Furthermore, we can assert that also the relation between continuity and change is dependent on the position of the observer. If we confine ourselves in a single epochal present - the domain of permanence of a specific field of relatedness - the temporal dynamics appears to us as not creative. If we place ourselves on a higher logical level where we take into consideration a process that contains more than one "epoch", change appears both creative and discontinuous, and shows the form of a succession of novel presents or fields of relatedness. From this standpoint is also possible to distinguish three forms of time: (i) as a succession of states in a single epochal present; (ii) as the duration of an epoch; and (iii) as a transformative discontinuous temporality that comprehends successive epochs.

\section{Conclusive Overview with Remarks on the Origins of Life}

The analysis of the concept of emergence in its unity - not just in its synchronic dimension - shows the limitations of our possibilities to describe the natural world, limitations that can concern the validity of our models both from the hierarchical and temporal points of view: two strictly co-dependent dimensions.

The thesis we sustained here is that the attempt to account in our conceptual models for the creativity of natural world, necessitates the assumption of a processual framework based on the notion of emergence, whose property consists in discontinuity. The concept of time it involves is not coherent with all the philosophical views on the creativity of nature. For example, in Bergson's thought ${ }^{42}$ the temporal dynamics is a 
unitary duration that grows thorough every moment by keeping its past. The evolutive dynamics deriving from the framework of diachronic emergence, instead, does not exhibit a unitary transformative temporality. Rather, at any temporal step it generates a new relational field that changes the identity of the process and, consequently, generates a discontinuity. Consequently, with regards to these aspects, this approach can be considered philosophically closer to Whitehead's idea of process as atomic and discontinuous. In fact it entails in any emergent step the generation of something new that revolutionizes the whole dynamics.

What is, then, the relationship between the epochal present and its past and future? The novel present is dependent on its past, from which it emerges. The past, in fact, is integrated in the new field of relatedness in the form of the constraints inherited by its previous steps. Examples are the chirality of biological macromolecules and the idea of "frozen accident" in evolution. Nonetheless, due to the nondeducibility condition, the derivative steps from present to past are precluded as well as those towards its future. In such a perspective, any temporal step cannot be considered as part of a linear chain, but rather like a node whose constitution reorganizes its past and future as horizons of possibilities. It provides constraints for the construction of possible past and future dynamics.

The domain of studies on the origins of life constitutes the emblematic example of the attempt to reconstruct the lost steps of a historical process. The idea of process we analyzed here, based on discontinuity and change of rules, has important consequences for the investigation in this field. In fact it collides with the principle of "continuity." This is the descendant of Lyell's principle of actualism in geology, one of the theoretical pillars of evolutionary gradualism, which asserts the need to explain the past by causes now in operation and, consequently, it excludes the investigation of possible different forces in act in different times. In some contemporary approaches to the issue of the origins of life, assuming its validity is considered as the only possibility to test scientifically our hypotheses on early evolution. ${ }^{43}$ Yet, this principle is not universally shared and has already been challenged both in geology and in biological evolution..$^{39,40}$ 
The idea of emergent processes, on the other side, entails the radical thesis according to which at any step in pre-biotic evolution there could have been a takeover by new forms of proto-life, replacing older ones and thus generating new contexts - new relational fields - by cancelling the previous ones. That such a takeover could have happened is even more probable if those processes were taking place in very small environments.

Surely, therefore, it is difficult to conceive the possibility of investigating the past if we cannot totally rely on the knowledge of the present. Maybe, it is still possible to think about tracing some of the characteristics of LUCA, which constitutes the first step of life as we know it. But the three of life is quite deeper and more complex. The image of a regular tree can be even misleading in this case, as it hides the possibility of takeovers. In fact, for this reason it is spreading as an alternative the use of the metaphor of the palm-tree of life, of which LUCA constitutes only the last segment, from which all present life evolved.

The idea of discontinuity seems to preclude the possibility of a scientific investigation of the previous segments of the trunk of the palmtree of life, cancelled by the succession of emergent steps and conserved only in the form of constraints. Nevertheless this conclusion is not totally true. A possible pathway of investigation is brought forth by some studies, like those in astrobiology and especially a part of synthetic biology, which attempt to create today new forms of pre-biotic evolution $^{44,45}$ without necessarily claiming that they are the true antecedents of contemporary life. What these researches do is, rather, to explore some of the possible pre-biological or early biological worlds which, by analogy, can provide useful information about a subset of the constraints that early evolution had to satisfy. As such, they do allow the formulation of some hypothetical scenarios about the history of life on earth. At the same time, they open new pathways for future evolution as well.

In conclusion, this framework entails a view of science that is alternative to the calculus of a linear chain of events, to the description of an algorithmic process from premises to consequences. It is in contrast with what Chaitin calls the "Software view of Science" 
scientific theory considered as a program that elaborates predictions about a world in which all the relevant information is already given as a input, and in which the activity of the scientist consists in identifying the right algorithm to describe it: a world and a science without emergences. Complex systems, by exhibiting logical openness, seem to escape this framework by putting into evidence the intrinsic limitations of our descriptive tools. As a consequence, in order to be approached they require a shift of attention from the refinement or the extension of a single model to the meta-domain of relations between different, and often complementary, models which work in the traditional way just inside their limited domain of validity. ${ }^{15,17}$ This means not just to choose which individual model is more appropriate in order to address a specific issue, but also to implement a "dynamical usage of models" 47,16 , that is, to take into consideration the interaction between more than one descriptive modality at the same time. A basic example can be found in the hybridization of bottom-up and top-down strategies in approaching the problem of protein folding.

This conceptual shift requires an anti-foundational perspective, where there is neither a primitive level of description nor constancy of laws to appeal to, but just an activity of investigation of natural phenomena through the construction of models that are limited in time and in the domain of application. This activity consists in the exploration of different temporal domains and possible worlds, of manifold hierarchical and logical levels, which are distinct but at the same time in a relation of mutual specification - through reciprocal selection and constraining and, as such, all necessary for the comprehension of the phenomena under study.

\section{References}

1. A. Lovejoy, in Proceedings of the Sixth International Congress of Philosophy, Ed. E. S. Brightman (Longmans, Green and Co, New York, 1927) p. 20.

2. J. Kim, Mind in a Physical World (MIT Press, Cambridge MA, 1998).

3. J. Kim, Physicalism, or Something Near Enough (Princeton University Press, Princeton, 2005).

4. S. Kauffman, Reinventing the Sacred (Basic Books, New York, 2008).

5. E. Nagel, The Structure of Science (Harcourt, New York, 1961). 
6. A. Stephan, Grazer Philosophische Studien, 65 (2002).

7. R. Rosen, Fundamentals of Measurement and Representation of Natural Systems (North-Holland, New York., 1978).

8. R. Rosen, Life Itself (Columbia University Press, New York, 1991).

9. L. Bich, L'ordine invisibile (Ph.D. Dissertation, University of Bergamo, 2008).

10. L. Bich, in Processes of Emergence of Systems and Systemic Properties, Ed. G. Minati, M. Abram and E. Pessa (World Scientific, Singapore, 2009), p. 591.

11. L. Bich, Synthese, forthcoming (2010).

12. H. Maturana and F. Varela, Autopoiesis and Cognition (Reidel Publishing, Dordrecht, 1980).

13. H. Maturana and F. Varela, El árbol del conocimiento (Editorial Universitaria, Santiago del Chile, 1984).

14. L. Damiano, Unità in Dialogo (Bruno Mondadori, Milano, 2009).

15. G. Minati, M. P. Penna and E. Pessa, Sys. Res.Behav. Sci., 15 (1998).

16. G. Minati and E. Pessa, Collective Beings (Springer, New York, 2006).

17. I. Licata, Epistemologia, 2 (2008).

18. R. Rosen, Anticipatory Systems (Pergamon Press, Oxford, 1985).

19. G. Kampis, Self-modifying Systems in Biology and Cognitive Science (Pergamon Press, Oxford, 1991).

20. S. Kauffman, Investigations (Oxford University Press, Oxford, 2000).

21. A. N. Whitehead, Science and the Modern World (Cambridge University Press, Cambridge, 1926).

22. A. N. Whitehead, Process and Reality (Macmillan, London, 1929).

23. E. Kraus, The Metaphysics of Experience (Fordham University Press, New York, 1994).

24. I. Stengers, Penser Avec Whitehead (Seuil, Paris, 2002).

25. M. Bitbol, Phenom. Cogn. Sc., 6 (2007).

26. H. Simon, The Sciences of the Artificial (MIT Press, Cambridge MA, 1969).

27. F. Jacob, La logique du vivant (Gallimard, Paris, 1970).

28. A. Vicente, Brit. J. Phil. Sci., forthcoming 2010.

29. M. Bitbol, Synthese, forthcoming (2010).

30. E. Pessa, The concept of particle in Quantum Field Theory (2009) http://arxiv.org/abs/0907.0178.

31. G. Minati, New Approaches for Modelling Emergence of Collective PhenomenaThe Meta-structures project (Polimetrica: Milano, 2008).

32. C. Lloyd Morgan, Emergent Evolution (Williams and Norgate, London, 1923).

33. J. K. Feibleman, Brit. J. Phil. Sci., 5 (1954).

34. P. K. Anokhin, Biology and Neurophysiology of the Conditioned Reflex (Pergamon Press, Oxford, 1974).

35. G. Egiazaryan and K. Sudakov, J. Hist. Neurosci., 16 (2007).

36. N. Baas, in Artificial Life III, Ed. C. Langton (Addison-Wesley, Reading, 1994), p. 515. 
37. G. Bocchi and M. Ceruti, Modi di pensare postdarwiniani (Dedalo, Bari, 1984).

38. G. Bocchi and M. Ceruti, Origini di storie (Feltrinelli, Milano, 1993).

39. G. Bocchi and M. Ceruti, Il fuoco e il gelo. Vincoli e possibilità della vita (forthcoming).

40. S. Gould, The Structure of Evolutionary Theory (Harvard University Press, Harvard MA, 2002).

41. H. Maturana and J. Mpodozis, Rev. Chil. Hist. Nat., 73 (2000).

42. E. Bergson, L'Evolution créatrice (Alcan, Paris, 1907).

43. J. Griesemer, in Philosophy of Biology, Ed. M. Ruse (Oxford University Press, Oxford, 2008) p. 263.

44. P. L. Luisi, F. Ferri and P. Stano, Naturwissenschaften, 93 (2006).

45. S. Rasmussen, M. Bedau, L. Chen, D. Deamer, D. C. Krakauer, N. H. Packard and P. F. Stadler, Eds., Protocells: Bridging Nonliving and Living Matter (Mitt Press, Cambridge MA, 2009).

46. G. Chaitin, Scientific American, March 2006.

47. G. Minati and S. Brahms, in Emergence in Complex, Cognitive, Social and Biological Systems. Ed. G. Minati and E. Pessa (Kluwer, New York, 2002), p. 41. 